\title{
Rectal cancer management in elderly patients: experience of a single Portuguese institution
}

\author{
Isália Miguel, MD, ${ }^{a}$ Ana Luis, MD, ${ }^{a}$ Ricardo Fonseca, MD, ${ }^{b}$ Luis d'Orey, MD, ${ }^{\mathrm{c}}$ Manuel \\ Limbert, MD, ${ }^{\mathrm{c}}$ Isadora Rosa, MD, ${ }^{\mathrm{d}}$ João P Silva, MD, ${ }^{\mathrm{d}}$ José Venâncio, MD, ${ }^{\mathrm{e}}$ Paula Pereira, \\ $\mathrm{MD},{ }^{\mathrm{f}}$ Luisa Mirones, MD, ${ }^{\mathrm{f}}$ Gonçalo Fernandez, $\mathrm{MD},{ }^{\mathrm{f}}$ and João Freire, $\mathrm{MD}^{\mathrm{a}}$
}

Departments of ${ }^{\mathrm{a}}$ Medical Oncology, ${ }^{\mathrm{b}}$ Pathology, ${ }^{\mathrm{c}}$ Surgery, ${ }^{\mathrm{d}}$ Gastroenterology, ${ }^{\mathrm{e}}$ Radiology, and ${ }^{\mathrm{f}}$ Radiotherapy, Instituto Português de Oncologia de Lisboa Francisco Gentil, EPE, Lisbon, Portugal

Background The incidence of rectal cancer increases with age, and older patients are more likely to have other chronic conditions that can affect outcome and tolerability of treatment.

Objective To evaluate retrospectively the influence of age and comorbidities in the management of rectal cancer.

Methods 59 patients aged 75 years and older with stage II-III rectal cancer who were treated during a 3-year period were in-

cluded in the study. Comorbidities were assessed using the Charlson Comorbidity Index (CCI) and the patients were divided into 2 groups based on their $\mathrm{CCl}$ scores: Fit (score of $0-1$ points) and Vulnerable (score of $\geq 2$ ). Primary endpoint was survival at 1 and 3 years.

Results The sample included 43 patients $(72.9 \%)$ in the Fit group and 16 patients $(27.1 \%)$ in the Vulnerable group. The most common comorbidities were myocardial infarction, diabetes, and chronic lung disease. One-year survival the same between the groups $(P=.330)$, but 3-year survival was lower in the Vulnerable group patients ( $83.7 \%$ vs $56.3 \%$, respectively; $P=.040)$. The rates of neoadjuvant chemo- and radiotherapy use and low anterior resection performance were the same between the groups. Colostomy closure was achieved more frequently in the Fit group compared with the Vulnerable group $(83.3 \%$ vs $55.6 \% ; P=.083)$. There was no difference in mean disease-free survival, grade 3-4 toxicity, and dose reduction between the groups.

Conclusions Comorbidity assessment should always be included in standard oncological management of elderly patients. Fit patients can be managed with standard treatment and may benefit from a conventional, more aggressive approach in their therapy.

$\mathrm{C}$ ancer is the main cause of death between the ages of 60 and 79 years in developed countries. More than half of all cancer deaths occur in patients who are older than 65 years. ${ }^{1}$ Colorectal cancer (CRC) is the third most common neoplasia in men and the second in women, ${ }^{2}$ and rectal cancer accounts for a third of all CRC cases. ${ }^{3}$ The incidence of rectal cancer increases with age and peaks at 80-84 years (135 new cases per 100,000 people/year). ${ }^{4}$

Surgical resection remains the curative treatment for localized rectal cancer. ${ }^{4,5}$ However, there is scant evidence on the best surgical approach for elderly patients, considering the heterogeneity of the comorbidities with which they present and that within this population, a patient's overall health status can range from very fit to very frail. ${ }^{4,5}$ Elderly patients are undertreated compared with younger patients and account for fewer surgical procedures with curative intention. ${ }^{4}$
Surgical options for localized rectal cancer are transanal excision and radical surgery. ${ }^{5}$ The radical approach includes low anterior resection (which preserves sphincter function) or abdominal-perineal resection with permanent colostomy. The choice between these treatment modalities relies on tumor stage, location, surgical technical capacity, and patient characteristics. ${ }^{6}$ Usually, elderly patients are treated with nonsphincter preserving procedures, with low anterior resection (LAR) being used less frequently than it is in younger patients. The reasons for this disparity could be patient choice, risk of postoperative complication, or surgeon preference. ${ }^{4}$

As part of the curative treatment for localized rectal cancer, neoadjuvant chemoradiotherapy (CHRT) or radiotherapy (RT) are recommended. ${ }^{1}$ However, in elderly patients these options are not often used or considered. ${ }^{4}$ Moreover, some studies have revealed that only a reduced number of elderly patients are treated with adjuvant chemotherapy or

Accepted for publication December 2, 2014. Correspondence: Isália Miguel, MD; imiguel@ipolisboa.min-saude.pt. Disclosures: The authors have no disclosures. JCSO 2015;13:8-13. C2015 Frontline Medical Communications. DOI 10.12788/icso.0100. 
radiotherapy, even when those treatments have proven to be effective in reducing recurrence. ${ }^{7,8}$

Although it is estimated that $35 \%-45 \%$ of patients with rectal cancer are older than 75 years, ${ }^{4}$ elderly patients are underrepresented on clinical trials and publications, thus making it difficult to extrapolate results to this population. Common problems for the recruitment of elderly patients into clinical trials include higher susceptibility to toxicity, age-related altered metabolism of chemotherapy, higher comorbidities, lower life expectancy, and lack of social and financial support. ${ }^{9}$

Older patients are more likely to have other chronic conditions that can affect outcome and treatment tolerability, ${ }^{10}$ but advanced age alone should not be the only criterion to exclude effective cancer treatment that could improve a patient's quality of life or survival. ${ }^{1}$ When treating elderly patients with rectal cancer, comorbidities should be considered in order to select the most appropriate treatment regimen. ${ }^{1}$

Oncogeriatric assessment is useful to detect vulnerable elderly patients. The Charlson Comorbidity Index (CCI) is the most commonly used tool for comorbidity evaluation in cancer patients and it is an indicator of disease burden with a strong capability to predict mortality. ${ }^{11,12}$ Patient age and Charlson score were independent prognostic factors for survival in aged and older patients. The Charlson comorbidity score may be helpful in assessing outcome in elderly patients with rectal cancer. ${ }^{10}$

The aim of our retrospective study was to evaluate the influence of age and comorbidities in rectal cancer management and survival in a cancer center in Portugal.

\section{Methods}

We retrospectively evaluated elderly patients with stage II-III rectal carcinoma of a total of 371 patients with rectal cancer treated during January 2008-December 2010 at a cancer center in Portugal. We included patients aged $\geq 75$ years at the time of diagnosis. This age limit provided a population that has been associated with greater vulnerability to treatment toxicities, surgical complications, or permanent stoma in previous studies. ${ }^{4}$ Other inclusion criteria were histology-proven rectal cancer stages II-III with no distant metastasis assessed by abdominal and thoracic computerized tomography. All of the patients were assessed with transrectal ultrasonography and pelvic magnetic resonance.

Comorbidities were retrospectively assessed using the $\mathrm{CCI} .{ }^{13}$ The patients were divided into 2 groups: Fit (score, $0-1$ ) and Vulnerable (score, $\geq 2$ ). The standard of care chemotherapy regimens for rectal cancer in our institution include the following:

- Neoadjuvant CHRT: fluorouracil (5-FU) $225 \mathrm{mg} / \mathrm{m}^{2}$ per day during RT 50.4 Gy (5-6 weeks); in the pres- ence of comorbid conditions: short RT scheme 5 x 5 Gy (25Gy).

- Adjuvant chemotherapy: de Gramont regimen (5-FU bolus $400 \mathrm{mg} / \mathrm{m}^{2}+$ folinic acid $400 \mathrm{mg} / \mathrm{m}^{2}+5-\mathrm{FU}$ continuous $46-\mathrm{h}$ infusion $2,400 \mathrm{mg} / \mathrm{m}^{2}$ ) during 6 months.

- Alternative regimen with capecitabine $825 \mathrm{mg} / \mathrm{m}^{2}$ twice daily during RT was used when a central line was not in place.

Major toxicity was assessed and graded using the National Cancer Institute Common Toxicity Criteria v4.03. ${ }^{14}$ Follow-up consisted of carcinoembryonic antigen (CEA) and CA 19-9 measurement every 3 months, a thoracic-abdominal-pelvic computed-tomography (CT) scan every year, colonoscopy on the first and third year after treatment, and every 5 years thereafter. The primary endpoint was overall survival (OS) at 1 and 3 years. Secondary endpoints were disease-free survival (DFS), colostomy reversal rate, toxicity, and dose reduction.

Statistical analysis was performed using SPSS for Windows v17.0 (Chicago, IL). Chi-square and Fisher exact tests were used to compare frequencies between groups in categorical variables and Student $t$ test to compare means when the distribution was normal. We used Kaplan-Meier methods to assess survival and log-rank test (Cox-Mantel test) to compare survival between both comorbidity groups. A significance level of 5\% was assumed.

\section{Results}

\section{Patient characteristics}

During January 2008-December 2010, 59 elderly patients with stage II-III rectal carcinoma were treated at our institution (15.9\% of the total of rectal cancers treated). The main patient characteristics can be found in Table 1. Fortyseven patients $(79.7 \%)$ received neoadjuvant CHRT (5-FU regimen, 44 patients; capecitabine, 3) and 12 (20.3\%) had radiotherapy only (short scheme 25 Gy). Surgery was performed in 53 patients $(89.8 \%$; low anterior resection [LAR], 40; abdominoperineal resection, 12; transanal resection, 1). One LAR patient died in the early postoperative period. Adjuvant therapy was initiated in 20 patients (33.9\%; de Gramont, 18; capecitabine, 2). Colostomy closure procedure was done in 30 of 39 patients (76.9\%). Relapse occurred in 11 patients (18.6\%), local recurrence in 5 , and distant metastasis in 7 . The metastatic sites were liver ( 3 patients), lung and bone, 2 each; and adrenal gland and peritoneal carcinomatosis, 1 each.

In all, 43 patients (72.9\%) were classified as Fit on the basis of their CCI score (0-1) and 16 (27.1\%) were classified as Vulnerable $(\geq 2)$. Twenty-three patients had no comorbidities. The main comorbidities (see Table 2) were myocardial infarction (20.3\%), diabetes (16.9\%), and chronic lung disease (10.2\%).

One-year OS was $89.8 \%$ and 3 -year OS was $76.3 \%$. 
Mean OS was 31.64 (SD, 1.26) months (Confidence index [CI] 95\%, 29.17-34.10). One-year DFS was 84.7\% and 3-year DFS was 66.1\%. Mean DFS was 28.98 (SD, 1.35) months (CI 95\%, 26.29-31.68).

\section{Toxicities}

Fifteen patients (25.4\%) presented with grade 3 or 4 toxicities: gastrointestinal toxicities occurred in 10 patients (16.9\%), hematological in 3 (5.1\%), and cutaneous toxicity in 2 (3.4\%). Toxicities occurred after neoadjuvant CHRT in $21.3 \%$ and during adjuvant treatment in $25 \%$ of patients. Dose reduction during neoadjuvant CHRT was needed in 13 of 47 patients (27.7\%), and 1 patient discontinued treatment. During adjuvant treatment, the dose had to be reduced in 4 patients (20\%) and discontinued in 2 .

\section{Subgroup analysis}

Mean age between comorbidity groups was equal $(P=.398)$. Patients with lower and higher CCI scores were treated with neoadjuvant CHRT on the same proportion $(81.4 \%$ and $75 \% ; P=.416)$. In terms of sphincter preservation, low anterior resection was the selected procedure in $81.6 \%$ of patients in the Fit subgroup and $64.3 \%$ of those in the Vulnerable subgroup $(P=.267)$, and colostomy closure was achieved more frequently in the Fit subgroup $(83.3 \%$ vs $55.6 \% ; P=.083)$. There were no differences in the LAR rate $(P=.646)$ or colostomy closure $(P=.812)$ between patients who were younger or older than 80 years.

Patients with lower comorbidities presented more toxicity events ( $30.2 \%$ vs $12.5 \% ; P=.145)$, but dose reduction of CHRT or adjuvant CHT were equal $(P=.220$ and $P=.807$, respectively). Comorbidities did not affect the 1-year survival (93.0\% with Fit patients and $81.3 \%$ with Vulnerable; $P=.330)$ but there were differences on the 3 -year survival

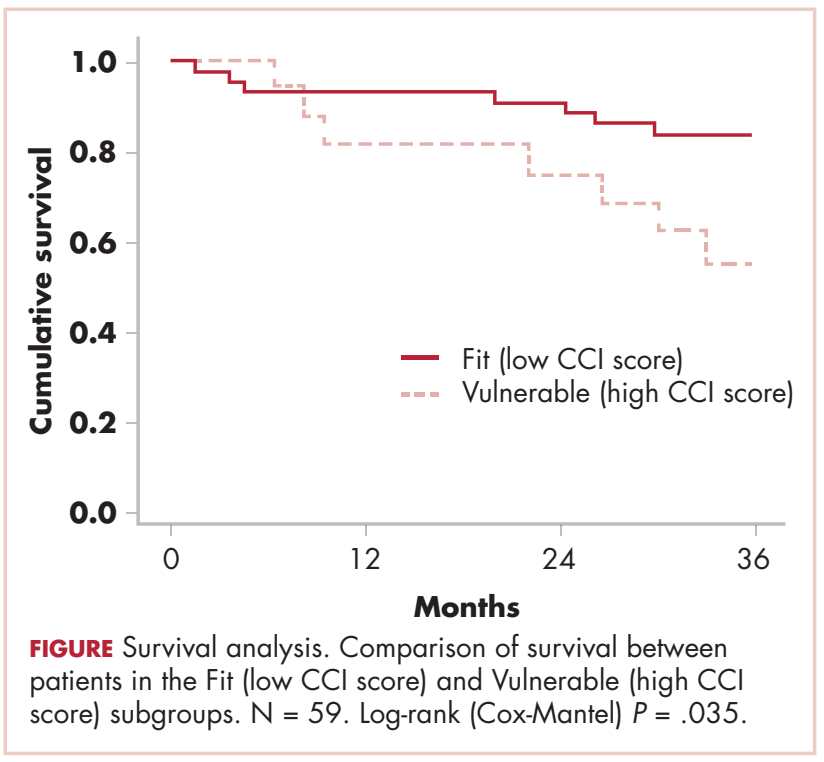

\begin{tabular}{|lc|}
\hline $\begin{array}{l}\text { TABLE } 1 \text { Patient characteristics }(N=59) \\
\text { Characteristic }\end{array}$ & \\
\hline Age group, $y$ & $33(55.9)$ \\
$75-79$ & $18(30.5)$ \\
$80-84$ & $8(13.6)$ \\
$\geq 85$ & \\
Gender & $39(66.1)$ \\
\hline Male & $20(33.9)$ \\
Female & \\
\hline Tumor location & $26(44.1)$ \\
\hline Low rectum & $25(42.4)$ \\
Middle rectum & $8(13.6)$ \\
Upper rectum & \\
\hline Preoperative T stage & $57(96.6)$ \\
\hline T3 & $2(3.4)$ \\
\hline T4 & \\
\hline Preoperative N stage & $20(33.9)$ \\
\hline N0 & $30(50.8)$ \\
N1 & $9(15.3)$ \\
\hline
\end{tabular}

TABLE 2 Scoring system and comorbidities for the Charlson Comorbidity Index ( $N=59$ )

\begin{tabular}{|c|c|c|}
\hline $\begin{array}{l}\text { No. of } \\
\text { points }\end{array}$ & Condition & $\begin{array}{c}\text { No. of } \\
\text { patients (\%) }\end{array}$ \\
\hline \multirow[t]{10}{*}{1} & Myocardial infarct & $12(20.3)$ \\
\hline & Congestive heart failure & $5(8.5)$ \\
\hline & Peripheral vascular disease & $5(8.5)$ \\
\hline & Cerebrovascular disease & $5(8.5)$ \\
\hline & Dementia & $2(3.4)$ \\
\hline & Chronic obstructive lung disease & $7(11.9)$ \\
\hline & Connective tissue disease & $0(0)$ \\
\hline & Peptic ulcer disease & $5(8.5)$ \\
\hline & Mild liver disease & $2(3.4)$ \\
\hline & Diabetes & $10(16.9)$ \\
\hline \multirow[t]{4}{*}{2} & Hemiplegia & $0(0)$ \\
\hline & Moderate/severe renal disease & $2(3.4)$ \\
\hline & Diabetes w/ end organ damage & $1(1.7)$ \\
\hline & Leukemia/lymphoma & $0(0)$ \\
\hline 3 & Moderate/severe liver disease & $0(0)$ \\
\hline 4 & AIDS & $0(0)$ \\
\hline 6 & Metastic solid tumor & $0(0)$ \\
\hline
\end{tabular}


TABLE 3 Comorbidity subgroup analysis $(N=59)$

\begin{tabular}{|c|c|c|c|c|}
\hline \multirow[b]{2}{*}{ Feature } & \multirow[b]{2}{*}{ Total } & \multicolumn{2}{|c|}{$\mathrm{CCl}$ subgroup } & \multirow[b]{2}{*}{$\boldsymbol{P}$} \\
\hline & & Fit $^{\mathbf{a}}$ & Vulnerable $^{b}$ & \\
\hline Mean age, y (SD) & $79.9(3.8)$ & $80.2(3.8)$ & $79.2(3.9)$ & $.398^{c}$ \\
\hline Neoadjuvant CHRT, \% & 79.7 & 81.4 & 75.0 & $.416^{d}$ \\
\hline Dose reduction, $\%$ & 29.8 & 34.3 & 16.7 & $.220^{d}$ \\
\hline Low anterior resection, \% & 75.5 & 81.6 & 64.3 & $.267^{\mathrm{d}}$ \\
\hline Colostomy closing, $\%$ & 76.9 & 83.3 & 55.6 & $.083^{d}$ \\
\hline 1-year overall survival, \% & 89.8 & 93.0 & 81.3 & $.330^{d}$ \\
\hline 3-year overall survival, \% & 76.3 & 83.7 & 56.3 & $.040^{d}$ \\
\hline Mean survival, mo. (SD) & $31.64(1.23)$ & $32.71(1.33)$ & $28.76(2.67)$ & $.035^{\mathrm{e}}$ \\
\hline
\end{tabular}

(83.7\% vs $56.3 \% ; P=.040)$ and mean survival (32.71 [SD, 1.33] vs 28.76 [2.67] months; $P=.035$ ), as seen in Table 3 and the Figure. Differences in mean DFS between both groups were not statistically significant (34.28 [0.82] and 31.09 [2.31] months; $P=.112$ ). The mean OS, 1 -year OS, or 3-year OS rates were not different in patients who were younger or older than 80 years $(P=.260, P=.077, P=.081$ respectively).

No gender-based differences were observed.

\section{Discussion}

Among cancer patients, elderly adults are the most prevalent age group but they are usually undertreated and excluded or underrepresented in clinical trials, which only include a minority of fit elderly patients. ${ }^{9}$ Almost all published results on the management of rectal cancer in elderly patients are based on retrospective series and on treatments that are not the standard of care. In colorectal cancer, it has been described that patients older than 70 years usually have nonmalignant comorbidities, most commonly, cardiovascular disease and hypertension. ${ }^{15}$ In our sample, cardiovascular disease was also the most frequently reported comorbidity, followed by chronic pulmonary disease.

In a recent systematic review on outcomes of rectal cancer surgery, investigators noted a dearth of data on rectal cancer management. ${ }^{4}$ They noted that the quality of most of the selected studies was poor, and most of them were single-center series and included aged adults with no comorbidities and intermediate functioning. ${ }^{4}$ Nevertheless, they emphasized that the presence of comorbidites, and not age, should be taken into account when considering curative surgical intention. Although data for curative surgery intent have shown an increased vulnerability of elderly patients and a higher risk of short-term mortality $(P<$ $.0001)$, there was no difference in 5-year relative survival after curative surgery between older and younger patients. Moreover, patient quality of life did not change with age. ${ }^{4}$

Neoadjuvant treatment for locally advanced rectal cancer is 5-FU-based chemoradiotherapy. The disadvantages of 5-FU continuous infusion include the placement of a central venous catheter, a portable pump, and restricted use in the presence of heart disease, which may have a negative impact on elderly patients. In our series, oral capecitabine instead of $5-\mathrm{FU}$ had to be administered in 3 patients because of central catheter placement problems.

Pasetto and colleagues have reported that tolerability of and response to chemoradiotherapy is the same in selected elderly "vulnerable" patients or "fit" old patients, ${ }^{9}$ but it is known that the presence of 3 or more comorbidities often leads to a decreased use of chemotherapy. ${ }^{16}$ Results from the Ontario Cancer Registry have revealed that patients with breast or colorectal cancer and a CCI score of $\geq 2$ were less likely to be treated with chemotherapy than were patients with a lower CCI score. ${ }^{5}$ In our sample, both comorbidity groups had a similar rate of CHRT use.

Preoperative radiotherapy alone remains controversial. It is known that secondary effects such as sexual dysfunction and fecal incontinence are more likely to occur in eldery patients, as the Stockholm I and II trials have shown. ${ }^{7}$ However, short-course, high-dose preoperative RT in phase 3 studies has been shown to increase the local control rate and disease-free survival compared with surgery alone. ${ }^{16,17}$ Our protocol reserves the short RT scheme for high-comorbidity patients who cannot be treated with CHRT.

The first choice surgical technique for rectal cancer in the elderly remains controversial. Data from the Swedish rectal cancer registry revealed that patients aged 75 years and older were less likely to have surgical tumor resection than were younger patients. Although it is known that tumor 
location does not differ between age groups, ${ }^{4}$ the rate of lower anterior resection was lower and nonrestorative procedures were performed more frequently than they were in the Swedish registry. ${ }^{8}$ In our results, more than $75 \%$ of surgical patients had an LAR, irrespective of age or comorbidity. Colostomy closure was done mainly in Fit patients of any age. Of the patients who were not offered closure, $55.6 \%$ had a low tumor location that could have interfered with functional results. In our series, 6 patients never had surgery, 3 patients refused surgery, and the rest had recently diagnosed comorbidities before surgery (myocardial infarction in 2 patients, and stroke in 1).

Colostomy closure should always be considered, and in our study, $76.9 \%$ of the patients had the procedure. Stoma reversal is obtained in 51\%-92\% of patients, according to previously reported single-institution and multi-institution studies of colostomy reversal among rectal cancer patients of all ages. ${ }^{18}$ Predictors of delay or failure in reversal include postoperative chemotherapy, increasing age, metastatic disease, comorbidities, and peri-operative complications. ${ }^{18}$ In a multivariate analysis, 2 independent factors for a permanent stoma were age greater than 75 years and symptomatic anastomotic leak. ${ }^{4}$ In our study, even though low-comorbidity patients were more likely to have the colostomy reversed than not ( 83.3 vs $55.6 \%$, respectively) it was not statistically significant, probably because of the smaller sample size. However, we observed no differences between old and very old patients.

They might be concerns about the functional results of restorative surgery in the elderly. Older adults are known to have deterioration on the pelvic diaphragm muscles and external anal sphincter function, and that can explain the preference for permanent colostomy procedures. Nevertheless, quality of life at this age could be more important than intestinal transit restoration. ${ }^{4}$ Two meta-analyses have shown that the overall quality of life was similar in patients who had had abdominoperineal resection compared with patients with intestinal continuity. ${ }^{19,20}$ Therefore, if colostomy closure is deemed impossible upfront, the surgical procedure can be simplified.

Adjuvant treatment aims to reduce disease recurrence and improve survival, but not many elderly cancer patients receive chemotherapy or radiotherapy although it has been shown to be effective., ${ }^{7,21,22}$ There is evidence that 5-FU-based adjuvant chemotherapy is associated with increased survival and decreased risk of relapse in all ages, ${ }^{16}$ which suggests that patients should not be denied adjuvant treatment because of age. ${ }^{7}$ The National Comprehensive Cancer Network's guidelines for treating rectal cancer suggest that leucovorin-modulated 5-FU, or FOLFOX (lecovorin+fluorouracil+oxaliplatin), or capecitabine, all with or without oxaliplatin, are appropriate options. ${ }^{23}$ Our data shows that adjuvant therapy (mainly de Gramont regi- men) was prescribed in $33.9 \%$ of patients. This is similar to published data of adjuvant $\mathrm{CT}$ use in the elderly, which accounts for $20 \%-51 \%$ of patients. ${ }^{15,21}$

Randomized studies have shown that 5-FU-based regimens are not associated with more toxicitiy in patients aged 70 years or older, except for those with leukopenia. ${ }^{5,19} \mathrm{In}$ our population, grade III-IV toxicities occurred in $25.4 \%$ of patients and were more frequent during adjuvant treatment and in the Fit subgroup (although not statistically significant), probably as a result of a more aggressive regime use.

The limitations of our study are its retrospective nature and the relatively small sample of patients because of the age limit in inclusion criteria. Nevertheless, we believe that patients older than 75 pose more challenges when it comes to deciding on treatment options and are often underreported in the literature. The fact that the current treatment protocol for rectal cancer in our institution was established in 2008 also limited the inclusion of previous cases and follow-up period.

\section{Conclusion}

With an ageing population, age alone should not be the reason for denying a full curative treatment in rectal cancer. Moreover, elderly patients should be managed by a multidisciplinary team to be able to recognize and improve the management of comorbidities and customize the best treatment option for the patient. In our experience, "fit" patients who are treated with neoadjuvant chemoradiotherapy and lower rectal surgery achieved a high rate of colostomy reversal and 3-year survival, which suggests they can be managed with standard treatment. For more "vulnerable" patients, a short course of radiotherapy followed by surgery seemed to have been adequate and was not associated with high toxicity. Prospective studies in this growing age group should be performed to assess the best treatment options.

\section{References}

1. National Comprehensive Cancer Network clinical practice guidelines in oncology. Senior adult oncology. Version 2.2014. In: www.ncen. org.

2. Labianca R, Nordlinger B, Beretta GD, et al. Early colon cancer: ESMO clinical practice guidelines for diagnosis, treatment and follow-up. Ann Oncol. 2013;24:64-72.

3. Glimelius B, Tiret E, Cervantes A, Arnold D. Rectal cancer: ESMO clinical practice guidelines for diagnosis, treatment and follow-up. Ann Oncol. 2013;24:81-88.

4. Manceau G, Karoui M, Werner A, Mortesen NJ, Hannoun L. Comparative outcomes of rectal cancer surgery between elderly and nonelderly: a systematic review. Lancet Oncol. 2012;13:e525-536.

5. Chang GJ, Skibbler JM, Feig BW, Rodriguez-Bigas M. Are we undertreating rectal cancer in the elderly? An epidemiologic study. Ann Surg. 2007;246:215-221.

6. Orsini RG, Thong MSY, van de Poll-Franse LV, et al. Quality of life of older rectal cancer patients is not impaired by a permanent stoma. Eur J Surg Oncol. 2013;39:164-70.

7. Bouvier AM, Jooste V, Bonnetain F, et al. Adjuvant treatments do not alter the quality of life in elderly patients with colorectal cancer. 
A population-based study. Cancer. 2008;113:879-885.

8. Jung B, Påhlman L, Johansson R, Nilsson E. Rectal cancer treatment and outcome in the elderly: an audit based on the Swedish rectal cancer registry 1995-2004. BMC Cancer. 2009;9:1-8.

9. Pasetto LM, Friso ML, Pucciarelli S, et al. Rectal cancer neoadjuvant treatment in elderly patients. Anticancer Res. 2006;26:3913-3924.

10. Cai X, Wu H, Peng J, et al. Tolerability and outcomes of radiotherapy or chemoradiotherapy for rectal cancer in elderly patients aged 70 years and older. Radiot Oncol. 2013;8:86.

11. Quan H, Li B, Couris CM, et al. Updating and validating the Charlson Comorbidity Index and score for risk adjustment in hospital discharge abstracts using data from 6 countries. Am J Epidemiol. 2011;173:676-682.

12. Lieffers JR, Baracos VE, Winget M, Fassbender K. A comparison of Charlson and Elixhauser comorbidity measures to predict colorectal cancer survival using administrative health data. Cancer. 2011;117:1957-1965.

13. Neugut AI, Fleischauer AT, Sundararajan V, et al. Use of Adjuvant chemotherapy and radiation therapy for rectal cancer among the elderly: a population-based study. J Clin Oncol. 2002;20:2643-2650.

14. National Cancer Institute. Common terminology criteria for adverse events (CTCAE). http://evs.nci.nih.gov/ftp1/CTCAE/CTCAE_4.03_2010-06-14_QuickReference_5x7.pdf. Updated June 14, 2010. Accessed December 11, 2014.

15. Kurtz JE, Heitz D, Serra S, et al. Adjuvant chemotherapy in elderly patients with colorectal cancer. A retrospective analysis of the implementation of tumor board recommendations in a single institution. Crit Rev Oncol Hemat.2010;74:211-217.
16. Dodgion CM, Neville BA, Lipsitz SR, et al. Do older Americans undergo stoma reversal following low anterior resection for rectal cancer? J Surg Res. 2013;183:238-245.

17. Charlson ME, Pompei P, Ales KL, MacKenzie CR. Anew method of classifying prognostic comorbidityin longitudinal studies: development and validation. J Chronic Dis. 1987;40:373-383.

18. De Marco MF, Janssen-Heijnen ML, van der Heijden LH, Coebergh JW. Comorbidity and colorectal cáncer according to subsite and stage: a population-based study. Eur J Cancer. 2000;36:95-99.

19. Cornish JA, Tilney HS, Heriot AG, Lavery IC, Fazio VW, Tekkis PP. A meta-analysis of quality of life for abdominoperineal excicios of rectum versus anterior resection for rectal cancer. Ann Surg Oncol. 2007;14:2056-2068.

20. Pachler J, Wille-Jorgensen P. Quality of life after rectal resection for cancer, with or without permanent colostomy. Cochrane Database Syst Rev. 2005;2:CD004223.

21. Swedish Rectal Cancer Trial. Improved survival with preoperative radiotherapy in resectable rectal cancer. N Engl J Med. 1997;336:980987.

22. Sebag-Montefiore D, Stephens RJ, Steele R, et al. Preoperative radiotherapy versus selective postoperative chemoradiotherapy in patients with rectal cancer (MRC CR07 and NCIC-CTG C016): a multicentre, randomised trial. Lancet. 2009;373:811-820.

23. National Comprehensive Cancer Network clinical practice guidelines in oncology. Rectal cancer. Version 3.2014. In: www.nccn.org.

24. Petersen SH, Harling H, Kirkeby LT, Wille-Jørgensen P, Mocellin S. Postoperative adjuvant chemotherapy in rectal cancer operated for cure. Cochrane Database Syst Rev. 2012;3:CD004078. 